\title{
La influencia de niveles básicos de procesamiento en la comprensión lectora de los alumnos sordos:Llevisión actual
}

\author{
Francisco Carrero Barril, Universidad de Málaga, Málaga, España
}

Resumen: En el presente estudio procederemos a revisar el papel que juegan los niveles inferiores de procesamiento lector en la comprensión cuando los alumnos sordos se enfrentan a los textos escritos. Los alumnos sordos presentan déficits en la competencia lingüistica general, lo que se traduce en déficit en las capacidades sintácticas y semánticas a nivel oral, lo que condiciona un abordaje del lenguaje escrito con dificultades de base. (Paul, 2009). Por otro lado, los alumnos sordos presentan déficits especificos en el procesamiento del lenguaje escrito (Marschark, 2008). En este estudio nos centraremos en los niveles que preceden a los procesos especificos de comprensión lectora. Revisaremos en profundidad tres de ellos: la decodificación fonológica, el acceso al léxico y el conocimiento del vocabulario. En nuestra revisión actual, nos centraremos en primer lugar en la relación entre los problemas de conciencia fonológica y los resultados en eficiencia lectora. Más adelante veremos las dificultades de los niños con déficit auditivo para el acceso a la palabra escrita y sus consecuencias en el acceso al significado del texto. Y finalmente, revisaremos si el volumen de vocabulario que el alumno sordo posee incide en el resultado final de la lectura.

Palabras Clave: Lectura, Comprensión, Sordos, Niveles, Procesamiento

Abstract: In the present paper, it is pretended to explain the role of basic levels in the processing of reading into the global comprehension when deaf students cope with writing texts. Deaf students have severe deficits in general language knowledge which involves deficits in syntactic and semantic abilities. And so they have several difficulties in reading and writing. Otherwise, deaf students have specific limitations in the processing of writing texts (Marschark, 2008). In the current review, the goal is to approach the specific difficulties in reading comprehension in the basis of processing: phonological awareness, lexical access and vocabulary knowledge. First, we will establish how phonological coding and reading efficiency are related. In the second hand, we asked if the limited lexical access influence final comprehension. Finally, it is noted that deaf students have a restricted vocabulary that affect their own ability to extract the global meaning of reading.

Keywords: Reading, Deafs, Phonological Coding, Lexical Access, Procesamiento

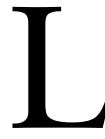

OS PROBLEMAS DE procesamiento en los alumnos sordos no sólo se circunscriben al procesamiento del lenguaje escrito. En el procesamiento del lenguaje oral nos encontramos con dificultades notorias que han sido ampliamente revisadas por la literatura científica en los últimos treinta años. En un estudio teórico clásico sobre diferencias cognitivas y lingüísticas entre alumnos sordos y oyentes (Lillo-Martin, 1997) se analizaron los resultados de varios estudios que examinaron las diferencias en diferentes tareas cognitivas entre oyentes y sordos, así como, entre sordos de padres sordos (con presumible exposición más temprana al lenguaje de signos) y sordos de padres oyentes. Los

Revista Internacional de Educaci ón y Aprendizaje

Volumen 1, 2013, http://sobrelaeducacion.com/revistas/coleccion/, ISSN 2255-453X

(C) Global Knowledge Academics. Francisco Carrero Barril

Todos los Derechos Reservados. Permisos: soporte@gkacademics.com

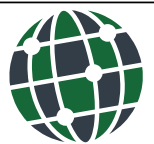


resultados indicaron que las diferencias entre sujetos oyentes y sordos procedían de las diferencias en los niveles inferiores del procesamiento (generación de imágenes, percepción visual y reconocimiento). Y se concluyó, además, que la exposición al lenguaje de signos en la infancia puede tener consecuencias diferentes en determinadas áreas del procesamiento cognitivo, pero estas diferencias de procesamiento no afectan directamente a los sistemas centrales, que implican un funcionamiento cognitivo de alto nivel.

Por lo tanto son los procesos de nivel inferior los que determinan las diferencias más significativas entre ambos grupos, al menos en el lenguaje oral. En el lenguaje escrito se ha trabajado mucho en los niveles básicos y los resultados han sido prometedores, especialmente en el caso de los procesos fonológicos (Leybaert \& Alegría, 2003), (Trezek, Wang, Woods, Gampp y Paul, 2007).

Un mayor porcentaje de los datos de que se disponen sobre comprensión lectora en sordos proceden de medidas de tests estandarizados. Wauters, Van Bon \& Tellings (2006) encontraron que los alumnos sordos de Educación Secundaria rindieron a un nivel equivalente de ocho años en un test similar. Con anterioridad, Traxler (2000) descubrió que los lectores con sordera de 18-19 años rendían a un nivel, lector equivalente al de niños de 8-9 años, un nivel medio ya encontrado en los estudios realizados en décadas anteriores.

Tales hallazgos han llevado a los autores de diferentes estudios a indagar sobre las razones por las cuales los alumnos sordos presentan este nivel deficitario en comprensión de textos escritos. Paul (2009) distingue tres grupos de factores que influyen de forma considerable: factores textuales, variables del propio lector y por último, variables de la tarea. Entre los factores textuales destacó la identificación de palabras, el vocabulario, la sintaxis y la presencia de lenguaje metafórico. Entre las variables del lector diferenció, entre otros, el conocimiento previo, la metacognición, la capacidad de memoria de trabajo y las habilidades de codificación fonológica. Y en cuanto a la tarea o contexto de la lectura tendrían notable influencia el objetivo de la lectura, el marco de la lectura y el tipo de medida de la comprensión lectora.

En nuestro estudio teórico nos centraremos en algunas variables textuales y del lector, haciendo hincapié en este último aspecto como fundamental en el acceso a la palabra escrita por parte del alumnado sordo. Profundizaremos a continuación en tres variables fundamentales para la comprensión y que forman parte de niveles de procesamiento inferior: conciencia fonológica o decodificación de unidades inferiores, identificación de palabras o acceso al léxico escrito y comprensión de vocabulario. Queda pendiente para una revisión posterior el complejo tema del procesamiento sintáctico de la lectura, que merece un capítulo aparte por su importancia teórica y práctica y por la extensión de la línea investigadora desarrollada en los últimos años.

\section{Codificación fonológica}

La codificación fonológica, relacionada de manera directa con las habilidades relacionadas con la conciencia de la fonología, es un factor fundamental en la comprensión de la lectura. Para los sordos, el acceso limitado al lenguaje oral puede dificultar el acceso a la información fonológica y, por tanto, a la habilidad de identificación de palabras. Los sordos, en general, elaboran una representación mental que puede considerarse funcionalmente equivalente al procesamiento fonológico utilizado por los lectores oyentes, pero muy diferente en su naturaleza esencial. Los estudios sobre el uso de la información fonológica en personas sordas 
han mostrado resultados muy dispares. Varios estudios han encontrado una relación significativa entre conciencia fonológica y tareas relacionadas con la lectura (Dyer et al., 2003), aunque otros estudios han fracasado al intentar contrastar esta relación (Kyle \& Harris, 2006). Parece existir una notable heterogeneidad en el acceso a la fonología, de tal manera que los resultados distan mucho de ser definitivos o concluyentes. Sí parece aceptado que los sordos con acceso al código fonológico presentan en los estudios unas características concretas: son mayores en edad, se muestran más habilidosos en la lectura, presentan mayor inteligibilidad en su habla y presentan mejores habilidades de labiolectura. El uso de la palabra complementada se ha conformado como un intento sistemático y riguroso para el acceso a la fonología, diseñado para convertir el habla en menos ambigua cuando las posiciones visuoespaciales y el lenguaje oral se muestran coarticulados y ha conseguido mejoras significativas en las habilidades lectoras para el idioma francés, pero no para el inglés (Leybaert \& Alegría, 2003).

Una forma de resolver el problema de la fonología sería a través de los fonos visuales. El sistema de fonos estaría conformado por 456 figuras de movimientos de la mano y símbolos escritos, utilizados simultáneamente con el lenguaje oral y la labiolectura, para representar aspectos de los fonemas de un lenguaje y su relación grafema-fonema. (Trezek, Wang, Woods, Gampp y Paul, 2007). Estos autores aseguran que los alumnos que utilizan esta herramienta mejoran en sus habilidades lectoras iniciales. No obstante no utilizaron grupo de comparación en Educación Infantil y Primer Grado, tan sólo en los cursos avanzados de Primaria, en los que sí se constató una evidente mejoría. Mientras se pulen estos estudios, parece ser que esta herramienta abre una nueva vía para enseñar a los sordos a leer de forma eficaz, un sistema que combina información fonológica y visual.

La relación entre fonología y ortografía conlleva procesos de abajo-arriba (guiado por los datos, por el texto) y de arriba-abajo (guiado por los esquemas del lector) al igual que el resto de niveles del procesamiento (identificación de palabras, procesamiento sintáctico, procesamiento semántico) por lo que su influencia en el acceso al significado del texto está presente durante todo el proceso lector.

\section{Acceso al léxico escrito}

Los estudios sobre habilidades para la identificación de palabras en los sujetos sordos han mostrado resultados variados y en ocasiones contradictorios. Wauters et al. (2006) encontraron escasas diferencias globales entre sordos y oyentes en tareas de identificación de palabras. No obstante, algunas diferencias se encontraron dentro de los grupos de edad, especialmente en los que estaban por debajo del nivel equivalente a $5^{\circ}$ de Primaria. Los estudios presentaban una alta heterogeneidad en variables tan relevantes como las características de los sujetos y las características de la tarea, por lo que se hace difícil extraer conclusiones definitivas sobre el rol que juega la identificación palabra en el aprendizaje de la lectura. Wauters et al. (2006) exhibieron puntuaciones de identificación de palabras que no fueron suficientes para dar cuenta de los déficits. La identificación de palabras y sus alteraciones no parecen ser suficientes para explicar las dificultades de comprensión lectora de los sordos. Aún cuando la identificación de palabras de los alumnos sordos no es inferior a la de los oyentes con deficiente comprensión o a la de los alumnos más pequeños con nivel similar, muestran niveles de comprensión lectora más deficientes que aquellos. 
Vermeulen, van Bon, Schereuder, Knoors \& Snik (2007) examinaron la relación entre identificación de palabra y comprensión lectora comparando dos grupos de edades variables, con o sin implante coclear. El grupo de implantados rindió mejor en tarea de comprensión, pero aún rindió por debajo que los oyentes de su grupo de edad normativo. Además, cuando la identificación de palabra fue estadísticamente controlada, las diferencias en comprensión lectora se mantuvieron, indicando que otras habilidades relacionadas con la lectura estaban contribuyendo a la mejora de las habilidades de acceso al significado de los sordos con implantes cocleares.

Parece claro, por tanto, que la identificación de palabra es una habilidad necesaria subyacente a la comprensión lectora y que interactúa notablemente con otras habilidades cognitivas y lingüísticas.

\section{Conocimiento del vocabulario}

El conocimiento del vocabulario es un predictor relevante de la comprensión lectora en las personas sordas (Kyle \& Harris, 2006). Los alumnos sordos han presentado habitualmente un volumen de vocabulario inferior a sus pares oyentes. Y desarrollan con menos facilidad procesos de adquisición de nuevos significados. Algunos autores han descubierto que la detección temprana de la pérdida auditiva conlleva un efecto especialmente positivo en el desarrollo del vocabulario (Lederberg, 2003). Los estudios sobre desarrollo de vocabulario en los sordos se han centrado en la relación entre vocabulario y lectura, teniendo en cuenta las características del alumno, de las cuales la presencia de implante coclear es una variable muy relevante a tener en cuenta.

Kelly (1996) se centró en la relación entre vocabulario y habilidad sintáctica en la lectura de los sordos y encontró que el vocabulario parecía ser un fuerte predictor de la comprensión lectora. Se encontró una fuerte relación con la tercera variable, la habilidad sintáctica, de tal manera que la relación vocabulario-comprensión lectora fue mayor en los sujetos con altas habilidades sintácticas que con respecto al grupo de bajas habilidades sintácticas. Garrison et als. (1997) estudió esta relación en sordos, añadiendo medidas de dos variables personales: conocimientos generales (conocimiento del mundo) e inferencias (estrategias de integración textual) del propio sujeto. Los sordos puntuaron, en general, a nivel inferior que sus pares oyentes y el vocabulario y el conocimiento del mundo fueron magníficos predictores para la comprensión lectora. Kyle \& Harris (2006) encontraron la relación entre vocabulario y comprensión lectora en jóvenes alumnos sordos, que rindieron significativamente peor que los oyentes. Wauters, Tellings van Bon \& Mak (2008) estudiaron la influencia en la comprensión de la modalidad de adquisición de los significados de las palabras. Las palabras que se adquirieron vía lenguaje fueron más difíciles de aprender y comprender, aun controlando su longitud y frecuencia, que las adquiridas a través de la percepción. Los resultados de comprensión fueron más bajos en los alumnos sordos (extremadamente bajos en las palabras adquiridas vía lenguaje). Aunque mejoraron los resultados en las palabras adquiridas a través de la percepción visual, la comprensión siguió siendo inferior en los sordos.

El vocabulario es un obstáculo relevante para el rendimiento lector de los alumnos sordos, lo cuales presentan una particular dificultad para las palabras más abstractas o para las adquiridas a partir de características lingüísticas, como más adelante veremos.

En la línea actual de investigación no es posible ya ninguna discusión sobre la relación entre comprensión lectora y el vocabulario, controlados los efectos del uso de implante 
coclear. Marschark, Rhoten y Fabich (2007) demostraron que, aunque los implantes cocleares conllevan una mejora en el aprendizaje de la lectura de los alumnos sordos, la ejecución de éstos siempre es más deficitaria que la de sus pares oyentes. Los resultados varían dependiendo de algunos factores como la edad de la implantación, la experiencia lingüística previa al implante coclear y el uso de la Comunicación Total.

En nuestra opinión sería aconsejable en un futuro realizar trabajos experimentales que pusieran en interacción las diferentes variables del procesamiento lector abordadas en las páginas anteriores. Y por supuesto llegar a conclusiones de mayor calado en cuanto a la relación entre cada una de ellas y la utilización adecuada del implante coclear, de cara a llegar a concusiones más definitivas acerca de las diferencias que pueden llegar a encontrarse entre ambos grupos de alumnos con discapacidad auditiva.

Por último reseñar la clara influencia que factores como la conciencia fonológica, la identificación de palabras escritas y el conocimiento de vocabulario, presentes en la lectura de los textos escritos (ya sean expositivos o narrativos) en el procesamiento semántico de la lectura, fase en la que podemos distinguir claramente dos componentes plenamente diferenciados: la creación del texto-base o sea la reproducción superficial del significado del texto mediante el código escrito y la construcción del modelo de la situación o modelo mental de la situación descrita por el texto, que representa el verdadero significado del texto (Kinstch \& Rawson, 2009).

\section{Conclusión}

Para una persona sin limitaciones perceptivas, motoras, cognitivas o lingüísticas, el acceso a la información escrita puede considerarse un proceso relativamente sencillo, dado el nivel de automatismo y eficacia que alcanzan un alto número de alumnos de Educación Primaria una vez que el aprendizaje de la lectura se ha desarrollado y consolidado de forma eficaz. La lectura, sin embargo, es una tarea muy laboriosa considerando la enorme cantidad de niveles y componentes que deben ser procesados de forma simultánea en un intervalo de tiempo reducido. Si el alumno presenta algún tipo de limitación, el proceso se convierte en una tarea aún más compleja. En el caso de los alumnos sordos, la lectura conlleva un amplio abanico de dificultades en distintos niveles que influyen de forma negativa para el acceso correcto a una comprensión adecuada de la información escrita, fin último del aprendizaje lector. A las ya consabidas limitaciones en lenguaje oral, hemos de añadir las dificultades que se van produciendo en los niveles inferiores del procesamiento lector (identificación de palabras, comprensión de vocabulario) que dejan para los procesos de alto nivel jerárquico, estrictamente orientados a la comprensión del texto, una mínima cantidad de recursos disponibles. No obstante, la hipótesis de la limitación de recursos no es suficiente para explicar de forma exclusiva el problema del acceso al significado. Aún cuando todos estos procesos relativos al lenguaje oral y escrito han sido experimentalmente controlados, se registran déficits concretos en las estrategias de comprensión, incluso en el caso de los alumnos con implante coclear con un desempeño cognitivo y lingüístico equivalente al de los alumnos oyentes.

Una de las escasas certezas que podemos sostener tras esta revisión es que el acceso temprano a un código lingüístico predice un aprendizaje lector superior. La adquisición del código lingüístico formal (oral o signado) proporciona al lector un contexto para adquirir las habilidades cognitivas y lingüísticas necesarias para este dominio y posibilita un mayor 
conocimiento del mundo (conocimientos generales), que, como conocimiento previo, juega un papel fundamental para que el alumno sordo pueda crear representaciones correctas acerca de los textos que lee y por tanto pueda acceder con menor dificultad a su significado. 


\section{Referencias}

Dyer, A ., MacSweeney, M ., Szczerbinski, M ., Green, L ., Campbell, R . (2003). Predictors of reading delay in deaf adolescents: the relative contributions of rapid automatized naming speed and phonological awareness and decoding. Journal of Deaf Studies and Deaf education, 8, 215-229.

Garrison, W. Long, G. \& Dowaliby, F. (1997). Working Memory Capacity and Comprehension Processes in Deaf Readers. Journal of Deaf Studies and Deaf Education, 2, 78-94.

Jackson, D. W., Paul, P. V., \& Smith, J. C. (1997). Prior knowledge and reading comprehension ability of deaf adolescents. Journal of Deaf Studies and Deaf Education, 2, 172-184.

Kelly, L. (1996). The interaction of syntactic competence and vocabulary during reading by deaf students. Journal of Deaf Studies and Deaf Education, 1, 76-90.

Kyle, F.E. \& Harris, M. (2006). Concurrent correlates and predictors of reading and spelling achievement in deaf and hearing school children. Journal of Deaf Studies and Deaf education, 11, 273288.

Lederberg, A. (2003). Expressing meaning. From communicative intent of building a lexicon. In M. Marschark, M. \& P.E. Spencer, (Eds). Oxford handbook of deaf studies, language and education (pp.247-260) New York: Oxford University Press.

Leybaert, J. \& Alegría, J. (2003). The role of cued speech in language development of deaf children. In M. Marschark, M. \& P.E. Spencer, (Eds). Oxford handbook of deaf studies, language and education (pp.262-274) New York: Oxford University Press.

Lillo- Martin (1997). The acquisition of English by deaf signers: Is Universal Grammar involved? In Flynn, S., Martohadjono, G. \& O'Neil, W. (Eds). The generative study of second language acquisition (pp.131-149). New Jersey. Lawrence Erlbaum Associates.

Marschark, M \& Wauters, L. (2008). Language comprehension and learning. In M. Marschark, M. \& P.C. Hauser, P.C. (Eds). Deaf cognition: Foundations and outcomes (pp 309-350). New York: Oxford University Press.

Marschark, M Rhoten C y Fabich M (2007). Effects of cochlear implants on children's reading and academic achievement. Journal of Deaf Studies and Deaf Education, 12, 269-282

Marschark, M., Lang, H. G., \& Albertini, J. A. (2002). Educating deaf students: From research to practice. New York: Oxford University Press.

Marschark, M., Sapere, P., Convertino, C., Seewagen, R. \& Maltzan, H. (2004). Comprehension of sign language interpreting. Journal of Deaf Studies and Deaf Education, 4, 345-368.

Marschark, M., Sapere, P., Convertino, C.M. \& Pelz, J. (2008). Learning via direct and mediated instruction by deaf students. Journal of Deaf Studies and Deaf Education, 13, 446-461.

Paivio, A. (2008). How children learn and retain information: The dual coding theory. In S. B. Newman (Ed.), Literacy achievement for young children in poverty. Baltimore: Paul H. Brookes.

Paul, P. (2009). Language and deafness. San Diego, CA: Singular Publishing Group.

Pinhas, J. (1991). Constructive processing in skilled deaf and hearing readers. In D. Martin (Ed.), $A d$ vances in cognition, education, and deafness (pp. 296-301). Washington, DC: Gallaudet University Press.

Richardson, J. T. E., McLeod-Gallinger, J., McKee, B. G., \& Long, G. L. (2000). Approaches to studying in deaf and hearing students in higher education. Journal of Deaf Studies and Deaf Education, 5, 156-173.

Strassman, B.K. (1997). Metacognition and reading in children who are deaf: a review of research. Journal of Deaf Studies and Deaf Education, 2,140-149.

Traxler, C.B. (2000). Measuring up to performance standards in reading and mathematics: Achievement of selected deaf and hard of hearing students in the national norming of the 9th Edition Stanford Achievement Test. Journal of Deaf Studies and Deaf education, 10, 256-271. 
Trezek, B., Wang, Y., Woods, D., Gampp, T., Paul, P. (2007). Using visual phonics to supplement beginning reading instruction for students who are deaf or hard of hearing. Journal of Deaf Studies and Deaf Education 12:373-384.

Vermeulen, A.M., van Bon, W., Schereuder, R., Knoors H., \& Snik, A. (2007). Reading comprehension of deaf children with cochlear implants. Journal of Deaf Studies and Deaf Education, 12, 283-302.

Wauters, L.N., Tellings, A.E.J.M., van Bon, W.H.J., \& Mak, W.M. (2008). Mode of Acquisition as a Factor in Deaf Children's Reading Comprehension. Journal of Deaf Studies and Deaf Education, 13, 176-192.

Wauters, L.N., Van Bon, W.H.J \& Tellings, A.E.J.M. (2006). Reading comprehension of Dutch deaf children. Reading and Writing: An interdisciplinary journal, 19, 49-76.

\section{Sobre el Autor}

Francisco Carrero Barril: Licenciado en Psicología. Master en Psicología del Lenguaje. Profesor en la Universidad de Málaga desde 2003. Experiencia docente en Trastornos del Desarrollo del lenguaje e Intervención en los Trastornos del Espectro Autista, la Parálisis Cerebral, el Trastorno del Desarrollo Intelectual y Trastornos del habla infantil. En Diplomatura de Magisterio en Audición y Lenguaje y Diplomatura y Grado de Logopedia. He participado en 2 proyectos de innovación educativa centrados en el trabajo en equipo en atención a al diversidad (2008-10 y 2010-12) Numerosas publicaciones y contribuciones a congresos derivadas de los proyectos de innovación y de experiencias de innovación de la propia docencia. En la actualidad participo en el proyecto de investigación EDU2011-26268 "El efecto de los avances audiológicos en el desarrollo del lenguaje y el aprendizaje escolar de los alumnos sordos". En proceso final, realización de trabajo experimental sobre "Modelos de situación en la comprensión lectora de las personas sordas". 